\title{
The Molecular Structures of Poly(3-hexylthiophene) Films Determine the Contact Properties at the Electrode/Semiconductor Interface
}

\author{
Yeong Don Park \\ Department of Energy and Chemical Engineering, Incheon National University, Incheon 406-772, Korea \\ E-mail: ydpark@incheon.ac.kr \\ Received February 14, 2014, Accepted April 7, 2014
}

\begin{abstract}
The contact properties between gold and poly(3-hexylthiophene) (P3HT) films having either of two distinct molecular orientations and orderings were investigated. Thermal treatment increased the molecular ordering of P3HT and remarkably reduced the contact resistance at the electrode/semiconductor interface, which enhanced the electrical performance. This phenomenon was understood in terms of a small degree of metal penetration into the P3HT film as a result of the thermal treatment, which formed a sharp interface at the contact interface between the gold electrode and the organic semiconductor.
\end{abstract}

Key Words : Metal penetration, Transfer line method, Organic transistor, Contact resistance

\section{Introduction}

Organic field-effect transistors (OFETs) have drawn interest due to their many possible applications in light weight, large-area, flexible electronics. ${ }^{1-4}$ Poly(3-hexylthiophene) (P3HT) is a conjugated polymer which has received considerable attention because of its good electrical and optical characteristic when used as a semi-conducting material in OFETs. ${ }^{5-8}$ The use of this polymer also enables simple, low-cost processing because it is soluble and conducting. ${ }^{9}$ These characteristics make P3HT an attractive candidate material for use in inexpensive flexible displays.

Despite its advantages, P3HT's utility has been hampered by its low field-effect mobility and high operating voltage in devices based on solution-processed conjugated polymer films. One approach to overcoming these drawbacks involves the development of well-ordered interconnected nanowires that provide good overlap between the $\pi$-orbitals of the conjugated organic molecules, thereby enabling efficient charge migration. ${ }^{10,11}$ Spin-coating methods, which can be used to produce homogeneous films, cannot be used to produce highly crystalline films because rapid solvent evaporation during film formation creates kinetically unfavorable conditions for crystal growth. ${ }^{12}$ In an effort to produce molecularly well-ordered structures that yield favorable electrical properties, researchers have investigated the effects of postprocessing treatments, such as thermal or solvent annealing. ${ }^{13-17}$

Many research groups have reported that improved the field-effect mobility of P3HT through post-treatments that promote the formation of self-organized conjugated lamellae and increase two-dimensional transport. ${ }^{18-21}$ Cho's group report that depending on the surface propertied, the P3HT nanocrystals can adopt two different orientations-parallel and perpendicular to the substrate-which have field-effect mobilities that differ by a factor of $4 .{ }^{20}$ Bao's group syste- matically characterized the behavior of $\mathrm{P} 3 \mathrm{HT}$ thin film both during the thermal annealing process and after thermal annealing. ${ }^{21} \mathrm{X}$-ray scattering and AFM about P3HT thin film suggest two distinct crystallization mechanisms that occur for annealing above and below $\mathrm{T}_{\mathrm{m}}$. De Leeuw's group reported that thermal annealing in vacuum performed on thin films grown on OFETs can eliminate unintentional doping for organic semiconductor. ${ }^{22}$ Many studies have investigated the effect of the thermal treatment on the crystallinity and the morphology, and also established the relation between nanoscale structured morphologies and electrical characteristics. But little is known about how the interface is stabilized and how the metal penetration at the interface may be optimized to obtain a higher mobility in a post-treated film. ${ }^{23}$ In fact, during OFET operation, charge carrier transport depends strongly on the interface properties. Key extracted device parameters, such as the carrier mobility, threshold voltage, and on/off current ratio are all affected by the presence of significant contact resistance. ${ }^{24}$ Charge injection at an electrode/semiconductor interface and the contact resistance can predominate over the channel resistance. The contact resistance becomes increasingly important in smaller devices by limiting the device charge carrier mobility and the transistor switching speed. To date, however, the interface between the conjugated polymer and the metal electrode has received little attention.

This study used the transfer line method to examine the contact resistance of the interface between gold and a P3HT layer having different molecular orientations and orderings. Although several studies have examined thermally treated P3HT films, few have addressed the impact of molecular structure on the contact resistance. Here, we explored the relationship between the molecular structural ordering and the contact properties. We found that the contact properties varied significantly in P3HT devices fabricated using different processing techniques. 


\section{Experimental}

Device Fabrication. P3HT obtained from Rieke Metals, Inc. (regioregularity $\sim 90 \%$, molecular weight, $M_{\mathrm{w}}=20-30$ $\mathrm{kDa}$ ) was used as received without further purification. The devices were fabricated on a heavily doped n-type silicon substrate as a common gate electrode. A thermally grown $300 \mathrm{~nm}$ thick $\mathrm{SiO}_{2}$ layer was employed as a gate dielectric (capacitance $=10.8 \mathrm{nF} \mathrm{cm} \mathrm{cm}^{-2}$ ). Two samples were prepared by spin-coating a $0.5 \mathrm{wt} \%$ regioregular $\mathrm{P} 3 \mathrm{HT}$ solution in chloroform $\left(\mathrm{CHCl}_{3}\right)$ onto silicon substrates (thickness of film: $\sim 32 \mathrm{~nm}$ ). One of the P3HT films was maintained at $240{ }^{\circ} \mathrm{C}$ for $30 \mathrm{~min}$ in a glove box and then cooled slowly. P3HT-based transistors were formed by evaporating gold through a shadow mask. The contact resistance was determined as a function of the channel length (L), which was varied from 100 to $250 \mu \mathrm{m}$, for a fixed channel width (W) 1 mm.

Characterization. Grazing incidence X-ray diffraction (XRD) studies were performed at the 3C beamlines of the Pohang Accelerator Laboratory (PAL), Korea. An atomic force microscope (AFM, Multimode IIIa, Digital Instruments) was used to characterize the surface morphologies of the samples. The electrical performances of the OFETs were characterized using a semiconductor analyzer (Keithley 4200) at room temperature. Gold was deposited at a rate of $0.5 \AA^{-1}$ in several steps in an ultrahigh vacuum system (base pressure $<1 \times 10^{-9}$ Torr) at submonolayer coverage levels on the P3HT substrates. Gold is a nonreactive material and so was chosen as the electrode in this study of the effect of metal penetration into the conjugated polymer. At each step, the interface between the gold and P3HT layers was characterized by measuring the $\mathrm{C} 1 \mathrm{~s}$ peak using synchrotron radiation photoemission spectroscopy at the $8 \mathrm{~A} 2$ beamlines of the PAL, Korea. The gold coverage was determined at each step using a thickness monitor.

\section{Results and Discussion}

The variations in the crystalline phase characteristics (i.e., the chain orientations and crystallinity) of the P3HT thin films after thermal treatment were characterized using synchrotron XRD measurements. Figure 1 shows the out-ofplane XRD pattern obtained from the P3HT films before and after thermal treatment at $240{ }^{\circ} \mathrm{C}$ (above the melting temperature of P3HT) for $30 \mathrm{~min}$. XRD measurements carried out on the as-spun P3HT thin film revealed a weak reflection at $2 \theta=5.5^{\circ}$ corresponding to a $16.0 \AA$ spacing in the (100) plane without multiorder $(h 00)$ peaks, The out-of-plane (010) reflection due to $\pi-\pi$ interchain stacking (3.77 spacing), on the other hand, was strong, as shown in Figure 1. ${ }^{25,26}$ The Bragg peaks obtained from the thermally treated P3HT thin films, (100), (200), and (300), were surprisingly high in intensity, and the (010) reflection was not observed. The presence of $(h 00)$ reflections alone without other $(h k l)$ reflections indicated that the melt-crystallized P3HT has the edge-on structures with lamellae and the $\pi-\pi$ stacking (a)

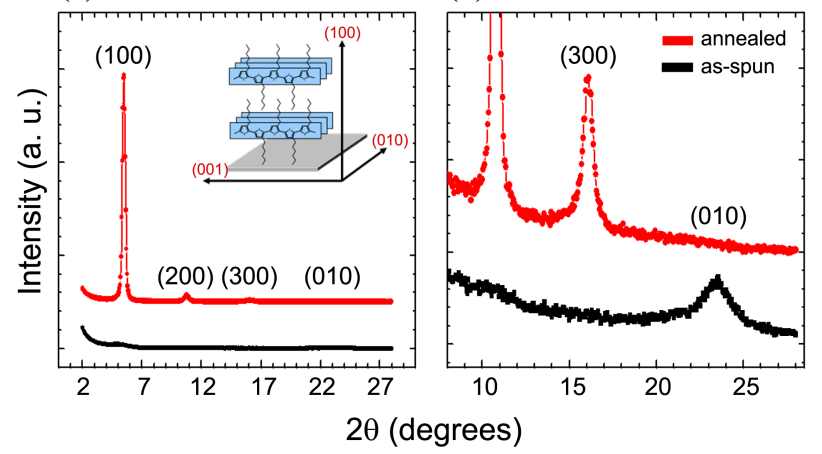

Figure 1. (a) Out-of-plane grazing incidence angle XRD intensities obtained from P3HT thin films on $\mathrm{SiO}_{2} / \mathrm{Si}$ substrates. (b) Magnified profiles of the (300), and (010) peaks in each scan direction. Inset shows schematic representation of the edge-on structure in P3HT.

direction preferentially oriented parallel to the substrate. We concluded that the thermally treated P3HT film had a much higher crystallinity than the as-spun film having a face-on structure with a (010) axis oriented normal to the substrate. Thermal treatment processes above the melting temperature $\left(\sim 240^{\circ} \mathrm{C}\right)$ induced re-orientation and reduced the residual stress that evolved during the spin-coating process.

Figures 2(a) and 2(b) show the AFM phase images of the P3HT thin films prepared without and with thermal treatment, respectively. The as-spun P3HT film appeared featureless. Interestingly, after thermal treatment by heating above the order-disorder transition temperature of the P3HT backbone chain $\left(240^{\circ} \mathrm{C}\right)$, rodlike microstructures $30-40 \mathrm{~nm}$ in width were observed in the phase image. Upon cooling the P3HT films from the melt after annealing above the $T_{m}$, heterogeneous nucleation at the substrate interface results in a recrystallization of the $\mathrm{P} 3 \mathrm{HT}$ with rod-like morphologies. It is notable that the thermally treated P3HT thin film showed a higher root-mean-square surface roughness $(1.22 \mathrm{~nm})$ than the as-spun film $(0.89 \mathrm{~nm})$. This result was attributed to the larger nanorods present on the thermally treated film surface. Changes in the inner structure of the P3HT film induced changes in the P3HT film surface morphology.

The relationship between the molecular structure and the electrical properties was explored by measuring the device (a)

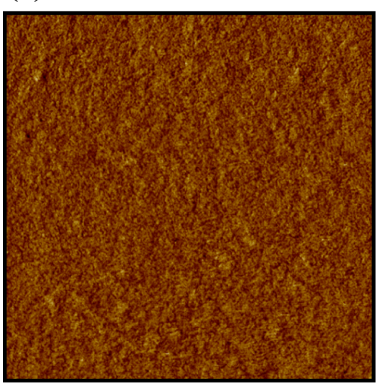

(b)

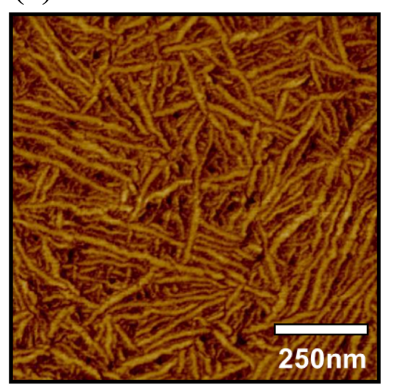

Figure 2. Tapping mode AFM phase images of the P3HT films: (a) As-spun, and (b) thermally treated P3HT. 
(a)
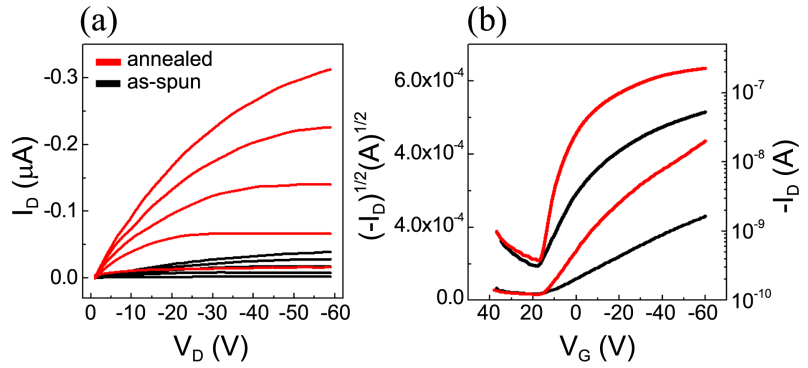

Figure 3. (a) Output characteristics $\left(\mathrm{I}_{\mathrm{D}} v s \mathrm{~V}_{\mathrm{D}}\right)$ of the FETs $(200$ $\mu \mathrm{m}$ long and $1000 \mu \mathrm{m}$ wide) prepared using the as-spun thin films or thermally treated thin films. (b) Transfer characteristics $\left(\mathrm{I}_{\mathrm{D}} v s\right.$ $\mathrm{V}_{\mathrm{G}}$ ) of the P3HT FETs: as-spun thin film and thermally treated thin films.

performances using the top-contact geometry. Typical drain current $v s$. drain voltage plots in the devices with a channel length of $200 \mu \mathrm{m}$ at five different gate voltages are shown in Figure 3(a). The devices prepared using the thermally treated P3HT showed higher drain currents than those prepared using pristine $\mathrm{P} 3 \mathrm{HT}$ for a given drain voltage. Figure 3(b) shows representative transfer characteristics of the pristine spin-coated P3HT FETs and the thermally treated devices. The thermal treatment process improved the performance parameters of all P3HT FETs, including the field-effect mobility and on/off current ratio, relative to the parameters obtained using pristine P3HT films.

The typical performance of an as-spun P3HT device was characterized by an average field-effect mobility of $3.2 \times$ $10^{-4} \mathrm{~cm}^{2} \mathrm{~V}^{-1} \mathrm{~s}^{-1}$ and an on/off current ratio of $10^{2}$. This relatively low field-effect mobility mainly resulted from the poor crystallinity and the unfavorable orientations in the conjugated plane, which resulted from a low regioregularity ${ }^{27}$ and a rapid solvent evaporation rate during the spin-coating process. In contrast, the thermally treated P3HT device showed an average field-effect mobility of $3.5 \times 10^{-3} \mathrm{~cm}^{2}$ $\mathrm{V}^{-1} \mathrm{~s}^{-1}$ in the saturation regime $\left(V_{\mathrm{D}}=-60 \mathrm{~V}\right)$, a value that was about ten times the value obtained from a device based on the as-spun P3HT, as shown in Figure 4(a).

The contact effects of the source and drain electrodes, which influenced the carrier mobility, were quantified by measuring the channel length-dependence of the resistance at small drain voltages through an analysis of the $I-V$ characteristics, as shown in Figure $4(\mathrm{~b})$. The contact resistance $\left(R_{\mathrm{c}}\right)$ was obtained from the $L=0$ intersection of the measured device resistance at each gate voltage $\left(V_{\mathrm{G}}\right)$, as indicated by Eq. (1) ${ }^{28}$

$$
R_{\text {total }}=R_{c}+\frac{L}{W\left[\mu_{i} C_{i}\left(V_{G}-V_{T}\right)\right]},
$$

where $\mu_{i}$ is the intrinsic field-effect mobility and $V_{\mathrm{T}}$ is the threshold voltage. $R_{\text {total }}$ was obtained from the inverse slope of the $I-V$ curves in the linear regime.

At drain voltages $\left(V_{\mathrm{D}}\right)$ much lower than the gate voltage, the drain current followed a highly linear behavior, indicating ohmic contact between P3HT and the evaporated gold

(a)

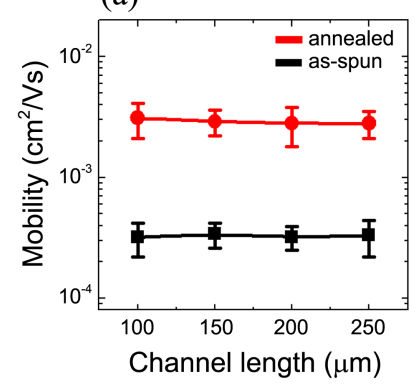

(b)

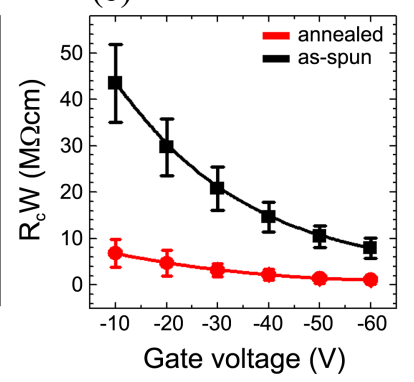

Figure 4. (a) Field-effect mobility obtained in the saturation regime for the as-spun and thermally treated P3HT FETs. (b) Channel width normalized contact resistance as a function of gate voltage.

electrodes. The total resistance, $R_{\text {total }}$, normalized by the channel width, was plotted as a function of the channel length $(L)$ for each $V_{\mathrm{G}}$ value, and the contact resistance of the evaporated gold electrodes was extracted from this plot. The channel-width-normalized contact resistance of pristine P3HT decreased from 44 to $7.9 \mathrm{M} \Omega \mathrm{cm}$ as $V_{\mathrm{G}}$ was reduced from -10 to $-60 \mathrm{~V}$ (Figure 4(b)). By contrast, the contact resistance of the thermally treated P3HT varied from 6.8 to $1.1 \mathrm{M} \Omega \mathrm{cm}$. A sevenfold decrease in the contact resistance of the device was observed upon thermally treating the P3HT thin film. This result may explain the higher mobility observed in the thermally treated P3HT device compared to the pristine device. The reduced contact resistance at the interface between the metal electrode and the P3HT upon thermal treatment played an important role in enhancing the device performance. ${ }^{29}$

The origin of the contact resistance reduction after thermal treatment was explored by examining the metal penetration into the as-cast and thermal treated P3HT film using X-ray photoemission (XPS) techniques. The relative $\mathrm{C}$ 1s peak intensity obtained from the XPS spectra after gold deposition onto the as-spun and thermally treated P3HT films is shown in Figure 5(a). The C 1s peak underwent a decrease upon deposition of the gold atoms onto the P3HT film. After deposition of the gold electrode onto the pristine film, how-

(a)

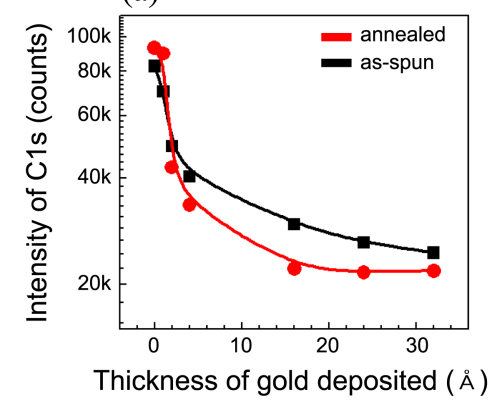

Figure 5. (a) Relative intensity of the $C$ 1s peak corresponding to gold deposited onto the as-spun and thermally treated P3HT films. Schematic views of the metal penetration on (b) an as-spun or (c) thermally treated P3HT film. The red lines indicate P3HT molecules and the yellow circles indicate gold particles. (b)
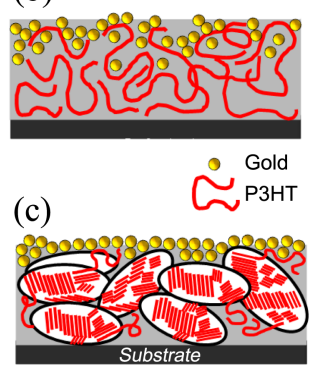
ever, the $\mathrm{C}$ 1s peak decreases slightly, indicating that gold atoms penetrated into the P3HT films in the amorphous region, and the $\mathrm{P} 3 \mathrm{HT}$ molecules migrated toward the air surface. After depositing the gold electrode onto the thermally treated film, the $\mathrm{C} 1 \mathrm{~s}$ peak intensity decreased significantly due to the attenuation effects of the deposited gold layer, indicating a low degree of metal penetration into the P3HT film.

The probability that a gold metal atom in a deposited electrode penetrates into the surface of a thermally treated P3HT film with a close packing density is smaller than the corresponding probability for an as-cast film. The thermally treated film therefore forms a sharper interface between the P3HT layer and the gold electrode (Figures 5(c)). A sharp interface between the organic semiconductor layer and the metal electrode reduces the contact resistance and yields a high field-effect mobility. ${ }^{18}$ Metal penetration into the asspun P3HT layer was more extensive and formed a broad interface in the contact region, as shown in Figures 5(b). The present results indicate that the performances of polymerbased transistors depended on both the molecular ordering of the semiconductor layer in the channel region and the contact properties of the interface between the electrode and the semiconductor layers, which indicates that the metal penetration is altered by changes in the molecular orientation and ordering of P3HT. The differences between the molecular ordering structures in the P3HT films provide an excellent opportunity for examining the fundamental properties of metal-organic interface formation in the context of metal electrodes and spin-coated polymer semiconductors.

\section{Conclusions}

In summary, we characterized the differences between the contact properties at the interfaces between a gold electrode layer and a P3HT film having one of two distinct molecular orientations and orderings. P3HT films that were thermally treated above the P3HT melting point assumed an ordered structure and an edge-on orientation. The field-effect mobility of a device prepared using a thermally treated P3HT thin film was found to be surprisingly greater than that of a device based on an as-cast P3HT film. The contact resistance was found to decrease by a factor of seven after thermal treatment of the P3HT film, possibly due to the formation of an abrupt interfacial contact structure between the metal electrode and the P3HT films. These results indicate that the contact resistance may be altered by changing the molecular orientations and orderings of a P3HT film.

Acknowledgments. This research was supported by Basic Science Research Program through the National Research Foundation of Korea (NRF) funded by the Ministry of Science, ICT \& Future Planning (2012R1A1A1004279). The authors thank the Pohang Accelerator Laboratory for providing the synchrotron radiation sources at $3 \mathrm{C}$ and $8 \mathrm{~A} 2$ beam lines used in this study.

\section{References}

1. Klauk, H. Chem. Soc. Rev. 2010, 39, 2643.

2. Facchetti, A.; Yoon, M. H.; Marks, T. J. Adv. Mater. 2005, 17, 1705.

3. Briseno, A. L.; Mannsfeld, S. C. B.; Shamberger, P. J.; Ohuchi, F. S.; Bao, Z.; Jenekhe, S. A.; Xia, Y. Chem. Mater. 2008, $20,4712$.

4. Ma, H.; Liu, M. S.; Jen, A. K.-Y. Polym. Int. 2009, 58, 594.

5. Park, Y. D.; Lim, J. A.; Lee, H. S.; Cho, K. Mater. Today 2007, 10, 46.

6. Torsi, L.; Magliulo, M.; Manoli, K.; Palazzo, G. Chem. Soc. Rev. 2013, 42, 8612

7. Kroon, R.; Lenes, M.; Hummelen, J. C.; Blom, P. W. M.; de Boer, B. Polym. Rev. 2008, 48,531

8. Kline, R. J.; McGehee, M. D. Polym. Rev. 2006, 46, 27.

9. Kang, B.; Lee, W. H.; Cho, K. ACS Appl. Mater. Interfaces 2013, $5,2302$.

10. Yang, H.; LeFevre, S. W.; Ryu, C. Y.; Bao, Z. Appl. Phys. Lett. 2007, 90, 172116.

11. Park, Y. D.; Lee, S. G.; Lee, H. S.; Kwak, D.; Lee, D. H.; Cho, K. J. Mater. Chem. 2011, 21, 2338.

12. Liu, J.; Sheina, E. E.; Kowalewski, T.; McCullough, R. D. Angew. Chem. Int. Eng. 2002, 41, 329.

13. Clarke, T. M.; Ballantyne, A. M.; Nelson, J.; Bradley, D. D. C.; Durrant, J. R. Adv. Funct. Mater. 2008, 18, 4029.

14. Park, Y. D.; Park, J. K.; Lee, W. H.; Kang, B.; Cho, K.; Bazan, G. C. J. Mater. Chem. 2012, 22, 11462.

15. Jimison, L. H.; Salleo, A.; Chabinyc, M. L.; Bernstein, D. P.; Toney, M. F. Phys. Rev. B 2008, 78, 125319.

16. Li, G.; Yao, Y.; Yang, H.; Shrotriya, V.; Yang, G.; Yang, Y. $A d v$. Funct. Mater. 2007, 17, 1636.

17. Quiles, M. C.; Ferenczi, T.; Agostinelli, T.; Etchegoin, P. G.; Kim, Y.; Anthopoulos, T. D.; Stavrinou, P. N.; Bradley, D. D. C.; Nelson, J. Nat. Mater. 2008, 7, 158.

18. Chen, F.-C.; Ko, C.-J.; Wu, J.-L.; Chen, W.-C. Sol. Energy Mater Sol. Cells 2010, 94, 2426.

19. Park, Y. D.; Kim, D. H.; Jang, Y.; Cho, J. H.; Hwang, M.; Lee, H. S.; Lim, J. A.; Cho, K. Org. Electron. 2006, 7, 514.

20. Kim, D. H.; Park, Y. D.; Jang, Y.; Yang, H.; Cho, K.; Kim, Y. H.; Han, J. I.; Moon, D. G.; Park, S.; Chang, T.; Chang, C.; Joo, M.; Ryu, C. Y. Adv. Funct. Mater. 2005, 15, 77 .

21. Verploegen, E.; Mondal, R.; Bettinger, C. J.; Sok, S.; Toney, M. F.; Bao, Z. Adv. Funct. Mater. 2010, 20, 3519.

22. Dinelli, F.; Murgia, M.; Biscarini, F.; de Leeuw, D. M. Synth. Met. 2004, 146, 373

23. Cho, J. H.; Kim, D. H.; Jang, Y.; Lee, W. H.; Ihm, K.; Han, J.-H.; Chung, S.; Cho, K. Appl. Phys. Lett. 2006, 89, 132101.

24. Chesterfield, R. J.; McKeen, J. C.; Newman, C. R.; Frisbie, C. D.; Ewbank, P. C.; Mann, K. R.; Miller, L. L. J. Appl. Phys. 2004, 95, 6396.

25. Park, Y. D.; Cho, J. H.; Kim, D. H.; Jang, Y.; Lee, H. S.; Ihm, K.; Kang, T. H.; Cho, K. Electrochem. Solid-State Lett. 2006, 9, G317.

26. DeLongchamp, D. M., Vogel, B. M.; Jung, Y.; Gurau, M. C.; Richter, C. A.; Kirillov, O. A.; Obrzut, J.; Fischer, D. A.; Sambasivan, S.; Richter, L. J.; Lin, E. K. Chem. Mater. 2005, 17, 5610.

27. Sirringhaus, H.; Brown, P. J.; Friend, R. H.; Nielsen, M. M.; Bechgaard, K.; Langeveld-Voss, B. M. W.; Spiering, A. J. H.; Janssen, R. A. J.; Meijer, E. W.; Herwig, P.; de Leeuw, D. M. Nature 1999, 401, 685.

28. Zaumseil, J.; Baldwin, K. W.; Rogers, J. A. J. Appl. Phys. 2003, 93, 6117.

29. Marinkovic, M.; Belaineh, D.; Wagner, V.; Knipp, D. Adv. Mater. 2012, 24, 4005 . 